\title{
Simulating Collective Transport of Virtual Ants
}

\section{Abstract}

This paper simulates the behaviour of collective transport where a group of ants transports an object in a cooperative fashion. Different from humans, the task coordination of collective transport, with ants, is not achieved by direct communication between group individuals, but through indirect information transmission via mechanical movements of the object. This paper proposes a stochastic probability model to model the decision-making procedure of group individuals and trains a neural network via reinforcement learning to represent the force policy. Our method is scalable to different numbers of individuals and is adaptable to users' input, including transport trajectory, object shape and external intervention etc. Our method can reproduce the characteristic strategies of ants, such as realign and reposition. The simulations show that with the strategy of reposition, the ants can avoid deadlock scenarios during the task of collective transport.

Keywords: Character Animation, Collective Transport

\section{Introduction}

Collective transport describes the behaviour of a group of ants collectively transporting a heavy prey, a task which would otherwise be impossible for a single individual to complete $[1,2]$. This cooperative behaviour saves the effort of dissecting a large prey on site and increases the overall amount of food supplied [2, 3]. Natural-looking animations of this behaviour could greatly enhance the vividness and immersion in interactive applications. However simulating the collective transport of virtual ants is a challenging task since it involves a group of individuals coordinating in an indirect way. It is even more challenging if the animator demands flexible control over the number of individuals, the trajectory, obstacles and other inputs.

In spite of the aforementioned challenges, few attempts have been made to model this behaviour in the field of computer animation. This deficiency is in sharp contrast with the large collection of existing work on simulating the interaction between biped characters $[4,5,6,7,8,9]$ and that of swarm behaviour $[10,11,12]$. Collective behaviour in humans normally requires intensive information sharing between individuals, such as in collaborative or adversarial games. Compared to such behaviours in humans, the collective transport of ants is not achieved by direct communication among individuals, but through indirect information exchange via the environment. This process is known as stigmergy [13]. Most of the existing work in swarm simulation focuses on navigation and formulation of swarm individuals and does not address the specific problem of force coordination in a decentralised scenario.

In this paper, we present a model for simulating the behaviour of collective transport of virtual ants. The goal of this work is not only to reproduce the phenomenon of collective transport, but also to allow animators to author sophisticated behaviours. The contributions of this work include:

- A novel stochastic probability model is introduced to simulate the strategies of $r e$ align and reposition, as used by ants during prey transport. This stochastic probability model produces the visually-appealing random behaviour by adjusting the ants' body orientation and attachment position during the process of collective transport.

- A stigmergy-inspired force policy is proposed and modelled as a neural network. 
The policy is further trained with the Qlearning method, a reinforcement learning technique, to optimise the weight parameters of the force policy network. With this force policy, characters can apply force to the object individually and successfully complete the task of collective transport without direct information from the others.

- We developed a complete framework to allow users to author the behaviour of collective transport. Our work is capable of scaling from two to a large number of individuals and can adapt to different scenarios based on user input of trajectories and prey weight etc. In the case of external intervention, individuals can reorganise themselves and restart the transport procedure.

The remainder of this paper is structured as follows. Section 2 surveys the existing work in related topics including multi-character interaction and swarm simulation. Section 3 describes the design of our framework. Section 4 presents the results generated from the proposed framework and discusses the limitations of our existing implementation. The last section, Section 5, concludes this paper by summarising and presenting directions for future research.

\section{Related Work}

\subsection{Multi-character Interaction in Computer Animation}

Recently there has been a surge of interest in modelling the interaction between multiple characters, in the field of computer animation $[4,5,6]$. Researchers initially focused on the interaction between two players by editing existing mocap data with an inverted pendulum model for each character [6], or by merging two existing interacting motion samples and automatically detecting the space-time relationship between them [5]. Game theory has been introduced to model the interaction of either collaborative or adversarial goals between two players $[7,8]$. Recent work has expanded to scenarios involving more than two characters. Based on written or verbal descriptions of the action scenes, researchers are capable of generating, ranking and recommending a small set of interaction scenarios for multiple characters from a large number of scene candidates [9]. Inspired by language grammars, researchers introduced a symbolic description to represent the interaction amongst individuals [4]. This has successfully generated animations for a group of characters in scenarios such as basketball games, where rules, regulations and planning are critical.

The complexity of the strategies, used in the existing work, far outperforms the intelligence of insects and is computationally unnecessary. Our work specifically focuses on the task of collective transport of ants and develops tools to simulate such behaviour with sufficient control over the group size, movement trajectory and more.

\subsection{Swarm Simulation}

143

Swarm simulation deals with the problem of ${ }_{144}$ generating the animation of a group of indi- ${ }_{145}$ viduals. Researchers introduced the concept of 146 navigation fields to direct and control virtual ${ }_{147}$ crowds [14]. These fields can be generated via 148 user sketches or 2D videos. Researchers have ${ }_{149}$ proposed interactive and scalable frameworks 150 which generate freestyle group formations and ${ }_{151}$ transitions via natural and flexible sketching in- 152 teraction $[10,11,12]$. Researchers have also ${ }_{153}$ proposed the control of sophisticated group for- ${ }_{154}$ mations via heuristic rules with explicit hard 155 constraints [15]. However, users had to man- 156 ually specify exact agent distributions, which 157 was time-consuming and labour intensive if the 158 crowd contained many agents. A recent work 159 [12] is capable of generating group behaviours 160 along with coherent and collision free naviga- 161 tion at interactive frame rates. Their method can 162 also dynamically adapt to the environment and ${ }_{163}$ the number, shape, and size of the groups. $\quad 164$

It is worth noting that there exists little work ${ }_{165}$ in the area of swarm simulation which ad- ${ }_{166}$ dresses the specific problem as proposed in this ${ }_{167}$ work. The majority of existing work focuses on 168 the distribution, navigation and formulation of 169 swarm individuals. However, the main interest 170 of our work is to coordinate the behaviour strat- 171 egy and force policy of group individuals with 172 indirect information sharing between them. $\quad 173$

Another critical application for simulating 174 
collective transport is the field of swarm robotics. Tasks which are challenging for a single robot, with limited capabilities, can be conducted by a group of robots. This not only allows for the flexibility of adapting to different tasks with different numbers of robots but also increases the system's robustness with sufficient tolerance of individual failures [3]. To implement such a function, robots can be coordinated in either a centralised [16] or decentralised [17] fashion. A centralised structure guarantees the optimal solution but suffers from an exponentially scaling complexity with regards to the number of individuals. A decentralised structure leads to a sub-optimal result but is scalable to a varying number of group individuals. However, compared to animation research, this research in robotics does not consider the synthesis of fullbody motion and prioritises stability over other factors.

\section{Methodology}

The behaviour engine defines the individual's collection of internal states and the rules for switching from one state to another. Intuitively, the behaviour engine is modelled as a Finite State Machine (FSM) (Figure 1a). A character has three states: search, approach and transport.

- Search. Characters are initialised at random positions in the scene. They individually search for the prey object by dynamically adjusting their movement direction. Characters can detect the existence of the prey if the distance is within a range of $2 \mathrm{~cm}$ (based on the observations of the species Pheidole crassinoda [18]). Once the prey object enters their sensory range, they switch to the state of approach.

- Approach. The character will approach directly towards the prey object once it is detected. The state of approach will terminate if a collision between the geometric shape of the prey and character is detected. In this case, the character switches to the state of transport.

- Transport. Once connected, individuals determine how to apply force to move the prey given the mechanical feedback from 221 the prey and other information (such as the 222 desired trajectory). The transport state is 223 subdivided into three strategies: standard, 224 realign and reposition. During the stan- 225 dard sub-state, the character doest not ad- 226 just its relative position with respect to the 227 object. Inspired by observations of real 228 ants, we propose a Stochastic Probability 229 Model to simulate two typical strategies 230 which ants adopt for collective transport: 231 realign and reposition.

When the character is in the state of either 233 search or approach, we directly specify the ve- 234 locity to manipulate the character's locomotion 235 and synthesise the full-body animation based on 236 a Central Pattern Generator control framework 237 [19]. The following paragraphs explain the two 238 main components of our work: the Stochastic 239 Probability Model as part of the behaviour en- 240 gine and the force policy to determine the drag- 241 ging force when the individual is attached to the 242 prey object.

\subsection{Stochastic Probability Model}

\subsubsection{Realign}

The strategy of realign alters the body orienta- 246 tion of the individual without releasing its hold 247 of the prey object [18] (Figure 1b). The in- 248 tuition is that the ant will attempt to align the 249 object with its own orientation so that the ant 250 can pull the object while walking backwards 251 $[1,3]$. When a single ant experiences difficulty 252 in pulling the prey object, it attempts to pull 253 from varying directions. The strategy of realign 254 tends to occur before reposition and much more 255 frequently than reposition [13]. 256

Various factors, including object weight, sur- 257 face friction and obstacle obstruction, can all 258 contribute to the resistance which ants experi- 259 ence during transport and thus triggers the strat- 260 egy of realign. Therefore, we choose the term of 261 transport velocity as an abstraction of the prey 262 movement. A score $P_{\text {realign }}$ is computed as:

$$
P_{\text {realign }}=\frac{1}{1+\exp \left(0.5-\frac{\left\|\vec{\nu}_{o}\right\|}{\left\|\vec{\nu}_{a}\right\|_{\max }}\right)}
$$

where $\vec{\nu}_{o}$ is the velocity of the object. $\left\|\vec{\nu}_{a}\right\|_{\max } \quad 264$ is the maximum moving speed of the virtual ant. 265 


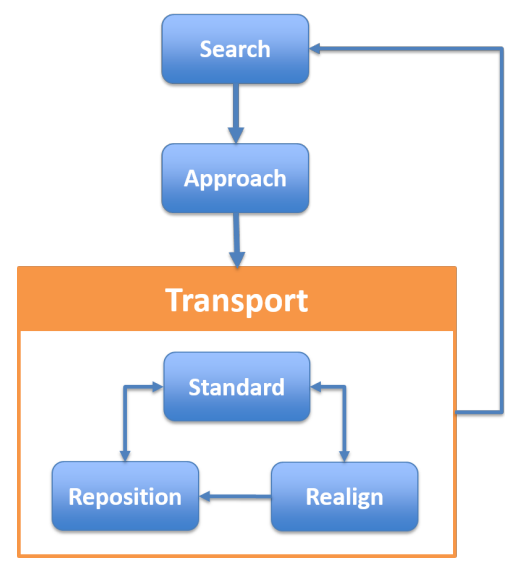

(a) FSM

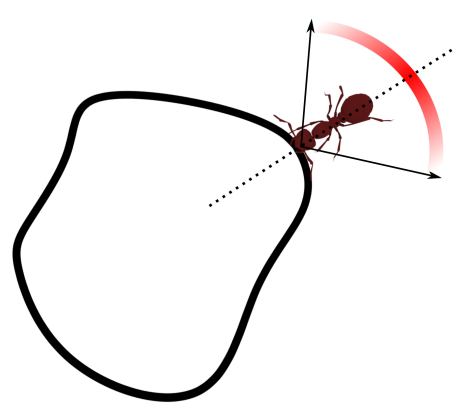

(b) Realign

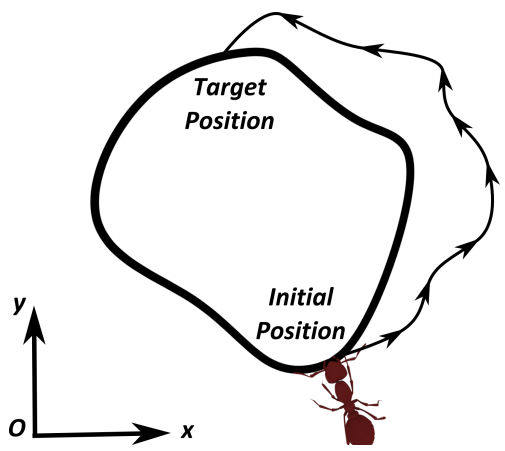

(c) Reposition

Figure 1: (a) Finite State Machine of the behaviour engine. (b) the strategy of realign. (c) the strategy of reposition.

If the individual still fails to move the prey after adjusting the pulling direction, it releases the attachment of the prey object, repositions itself at another attachment point and repeats the pulling process [18]. This process is called reposition (Figure 1c). A score $P_{\text {reposition }}$ is represented as following:

$$
\begin{aligned}
P_{\text {reposition }}= & \frac{1}{1+\exp \left(\frac{t}{t_{\max }}-\gamma\right)} \times \\
& \frac{1}{1+\exp \left(\frac{\left\|\vec{\nu}_{a}\right\|}{\left\|\vec{\nu}_{a}\right\|_{\text {max }}}-0.5\right)}
\end{aligned}
$$

where $\vec{\nu}_{a}$ is the movement velocity of the char- 284 acter. $t, t_{\max }$ are the elapsed time and the max- 285 imum time since the initialisation of current at- 286 tachment.

$P_{\text {reposition }}$ is also compared against a stochas- 288 tic threshold $\lambda_{p} \sim N\left(\mu_{p}, \sigma_{p}\right)$. Parameters 289 $\mu_{p}, \sigma_{p}$ are set to the same values as $\mu_{a}, \sigma_{a}$. If 290 the probability is greater than the threshold, the 291 character chooses to reposition itself, otherwise, 292 it does not.

The target reposition location is computed as a random point along the exterior shape of the object, which is uniformly parameterised between $[0,1]$. The movement trajectory $T(t)$ of reposition behaviour is computed as:

$$
T(t)=C_{o}(t)+D\left(t, D_{\min }, D_{\max }\right)+C_{c}(t)
$$

where $C_{o}(t)$ is the contour of the object shape in 299 the world coordinate, $D(t)$ is a uniform random 300 distribution between $\left[D_{\min }, D_{\max }\right]$, producing a 301 displacement distance between the character and 302 the object contour. $C_{c}(t)$ is a sub-level trajectory 303 to avoid the potential collision with other indi- 304 viduals. Our current implementation produces 305 $C_{c}(t)$ as a circular curve with a constant radius 306 and with its centre at the location of the other in- 307 dividual to avoid; although other types of curves 308 would also be suitable.

\subsection{Force Policy}

How the ants apply force to the prey object is 311 a challenging task, given the absence of com- 312 munication. We introduce a feed-forward neural 313 


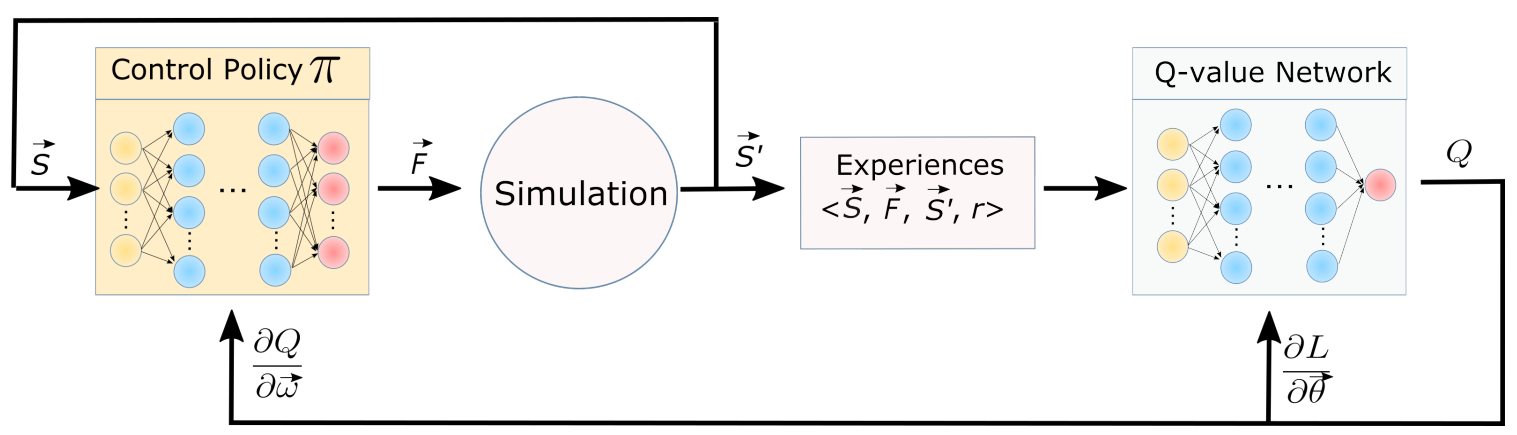

Figure 2: The framework of the force strategy. The control policy $\pi$ first determines the force $\vec{F}$ based on the current state $\vec{S}$. A Q-value network evaluates the performance of the control policy with a reward function.

network to define a policy $\pi$, which determines the force $\vec{F}$ applied on the object to change the current state $\vec{S}$ :

$$
\pi(\vec{S}, \vec{\omega}): \vec{S} \rightarrow \vec{F}
$$

where $\vec{\omega}$ is the parameters of the decision network. The input $\vec{S}=\left(\vec{\nu}_{a}, \vec{\nu}_{o}, \vec{\nu}_{o}^{*}\right)$ includes the velocity of the individual $\left(\vec{\nu}_{a}\right)$ and object $\left(\vec{\nu}_{o}\right)$, and the desired transport velocity of object $\left(\vec{\nu}_{O}^{*}\right)$.

We used the Q-learning method, a reinforcement learning algorithm, to optimise the parameters of the neural networks. In Q-learning, we first define a function $Q\left(\vec{S}, \vec{F}, \vec{\theta}_{Q}\right)$ representing the maximum discounted future reward when we choose $\vec{F}$ in state $\vec{S}$. We use a separate neural network to model the representation of the $\mathrm{Q}$ function and $\vec{\theta}_{Q}$ is the parameter of this second neural network.

The total future reward $Q$ is a sum of the rewards $r$ collected at each subsequent time step. The reward $r$ at a specific time is computed using:

$$
r=e^{-c_{\nu}\left(\vec{\nu}_{o}^{*}-\vec{\nu}_{o}\right)^{2}}+e^{-c_{\theta}\left(\theta_{b a c k}-\theta_{F}\right)^{2}}
$$

The first term minimises the difference between the actual and desired velocity, while the second term prioritises the backwards pulling direction. $c_{\nu}, c_{\theta}$ are positive constants for the respective terms. A data set $\left\langle\vec{S}, \vec{F}, r, \vec{S}^{\prime}\right\rangle$ is defined as an experience, which is collected and stored for latter training processes. $\vec{S}^{\prime}$ is the simulated state after the force $\vec{F}$ is applied in state $\vec{S}$.

Therefore, the $Q$ value for a specific time can be represented as:

$$
\begin{aligned}
Q_{t}(\vec{S}, \vec{F}) & =r_{t}+\gamma r_{t+1}+\cdots+\gamma^{n-1} \gamma_{t+n} \\
& =r_{t}+\gamma Q_{t+1}\left(\vec{S}^{\prime}, \vec{F}^{\prime}\right)
\end{aligned}
$$

\begin{tabular}{|c|c|c|}
\hline Layers & Force Policy & Q-value Network \\
\hline Input & 9 & 12 \\
\hline Hidden \#1 & 16 & 16 \\
\hline Hidden \#2 & 32 & 32 \\
\hline Hidden \#3 & 16 & 16 \\
\hline Output & 3 & 1 \\
\hline
\end{tabular}

Table 1: Architecture of the two neural networks used in this work.

where $\gamma$ is the discount factor of future reward. 342 $r_{t}$ is the reward at time $t$ computed by Equa- 343 tion 6. If $\gamma$ is zero, the policy only consid- 344 ers the instant reward and ignores the future re- 345 ward. When $\gamma$ is one, the policy considers the 346 full effect of future rewards even though they are 347 not deterministic. We choose a value (0.9) as a 348 reasonable balance between these two extremes. 349 Equation 7 is a Bellman equation, which means 350 that the Q-function can be approximated by iter- 351 atively updating this equation until convergence. 352

The force policy $\pi$ and Q-value network fol- 353 low a similar architecture design. Each network 354 is composed of 5 fully-connected layers. The 355 first and last layer are the linear-weight neurons. 356 The hidden layers are rectified linear units. The 357 number of neurons for each layer are listed in 358 Table 1.

The parameters $(\vec{\omega}, \vec{\theta})$ of the two neural net- ${ }_{360}$ works are optimised by the method of Stochas- 361 tic Gradient Descent (SGD). To iteratively opti- 362 mise the parameters of the Q-learning network, 363 we compute the loss function (or objective func- 364 
tion) using :

$$
L=\frac{1}{2}[\underbrace{r+\gamma Q\left(\vec{S}^{\prime}, \vec{F}^{\prime}, \vec{\theta}\right)}_{\text {target }}-\underbrace{Q(\vec{S}, \vec{F}, \vec{\theta})}_{\text {prediction }}]^{2}
$$

Therefore, the optimal gradient direction is:

$\frac{\partial L}{\partial \vec{\theta}}=\left[r+\gamma Q\left(\vec{S}^{\prime}, \vec{F}^{\prime}, \vec{\theta}\right)-Q(\vec{S}, \vec{F}, \vec{\theta})\right] \frac{\partial Q(\vec{S}, \vec{F}, \vec{\theta})}{\partial \vec{\theta}}$

For the control policy $\pi$, the optimal parameters of $\omega$ would produce the maximum reward $Q$. Therefore, the gradient of the optimal policy is the direction that most improves $Q$ :

$$
\frac{\partial Q}{\partial \vec{\omega}}=\frac{\partial Q}{\partial \vec{F}} \frac{\partial \vec{F}}{\partial \vec{\omega}}=\frac{\partial Q}{\partial \vec{F}} \frac{\partial \pi}{\partial \vec{\omega}}
$$

During runtime use, that is, after learning has been completed, the force is determined by forward-feeding the input through the decision network.

The force applied to the object is fundamentally related to the friction forces applied to the ant's stance legs. A double-tripod gait [20] is introduced to switch the legs between stance and swing. The front-left, middle-right and backleft legs are grouped as the Left Tripod while the other three legs are grouped as the Right Tripod. When the ant moves, the two groups of legs sequentially alternate between stance and swing. The following equations are used to distribute the desired dragging force $F_{i}$ among the stance legs:

$$
\begin{aligned}
{\left[\begin{array}{r}
m \ddot{\vec{p}} \\
\ddot{\ddot{\theta}}
\end{array}\right]=} & {\left[\begin{array}{ccc}
I^{D} & I^{D} & I^{D} \\
{\left[\vec{r}_{i, 1}\right]_{\times}^{T}} & {\left[\vec{r}_{i, 2}\right]_{\times}^{T}} & {\left[\vec{r}_{i, 3}\right]_{\times}^{T}}
\end{array}\right]\left[\begin{array}{c}
\vec{F}_{i, 1} \\
\vec{F}_{i, 2} \\
\vec{F}_{i, 3}
\end{array}\right] } \\
& +\left[\begin{array}{c}
-\vec{F}_{i}+\vec{G} \\
-\vec{F}_{i} \times \vec{r}_{i}
\end{array}\right]
\end{aligned}
$$

where $\vec{F}_{i, j}(j=1,2,3)$ is the force from the $j^{t h}$ stance leg of the $i^{\text {th }}$ individual. $I^{D}$ is the identity matrix. $m, I$ are the mass and inertia of the individual. $\vec{p}, \vec{\theta}$ are the position and orientation of the Center-of-Mass (COM) of each individual. $\vec{G}$ is the gravitational force. $\vec{r}_{i, j}=\left(r_{x}, r_{y}, r_{z}\right)$ is the vector connecting the $j^{\text {th }}$ footprint to the COM of the individual. $\left[\vec{r}_{i, j}\right]_{\times}^{T}$ is the corresponding skew-symmetric matrix of $\vec{r}_{i, j}$ :

$$
\left[\vec{r}_{j}\right]_{\times}^{T}=\left[\begin{array}{ccc}
0 & r_{z} & -r_{y} \\
-r_{z} & 0 & r_{x} \\
r_{y} & -r_{x} & 0
\end{array}\right]
$$

\begin{tabular}{|c|c|c|}
\hline Demonstration Task & $\begin{array}{c}\text { Number of } \\
\text { Characters }\end{array}$ & Frame Rate \\
\hline Deadlock (Figure 3) & 2 & 20.5 \\
\hline Crowd (Figure 5a) & 4 & 13.6 \\
\hline Crowd (Figure 5b) & 8 & 9.0 \\
\hline Crowd (Figure 5c) & 16 & 3.9 \\
\hline Crowd (Figure 5d) & 60 & 0.9 \\
\hline
\end{tabular}

Table 2: Experiment data of runtime performance of selected demos.

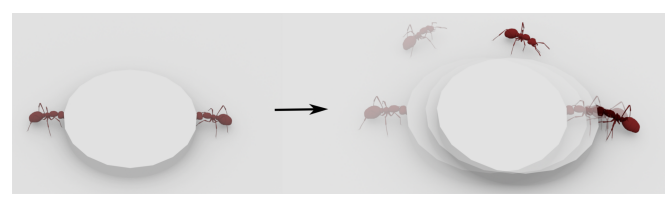

Figure 3: (Left) Using only the strategy of realign, two individuals can barely move the object. This creates the effect of deadlock. (Right) By repositioning one of the characters, two individuals apply force from a more consistent direction, thus resolving the issue of deadlock.

where $\vec{r}_{i}$ is the vector from the attachment point 384 to the COM of the individual. Equation 11 has 385 more than one solution if no further constraints 386 are introduced. We reduce the redundant dimen- 387 sions of the solution space by assuming that the 388 vertical forces are spread equally over the stance 389 legs.

\section{Results and Discussions}

The resulting motions from the behaviour en- 392 gine and the trained force policy, are best seen 393 in the supplemental video. The final force pol- 394 icy was resolved using 150k training iterations, 395 collecting about 1 million tuples. The complete 396 training process took approximately 30 hours on 397 an 8-core computer. We use the open source 398 deep learning framework Caffe [21] to build and 399 train the networks. The runtime performance 400 data, after training, is presented in Table 2. The 401 runtime data was collected on a standard laptop 402 with a Core i5-6200U @2.30GHz (CPU) and 403 8GB (RAM).

\section{8}

3
4
5
6
7
8
9
40
403
404




\subsection{Realign}

The strategy of realign adjusts the force direction applied by the individuals. In extreme cases (such as in Figure 3), two individuals drag the object from either ends, pulling the object in opposite directions. By adjusting the force directions only, the average translational velocity of the object is close to zero. In observations of real ants, the deadlock resulting from antagonistic pulling is rare and short in duration since real ants would soon reposition themselves [18]. To resolve this deadlock, one of the characters would choose to release the object and pick a new attachment point. This is illustrated on the right side of Figure 3.

\subsection{Reposition}

The strategy of reposition adjusts the point from which the individuals apply force. This strategy reduces the possibility of deadlock. This is further verified in the case of collective transport by a group of individuals (Figure 4). Six characters are initialised with even spacing around the object. Since the force policy is trained with the preference of dragging the object in a backwards direction, it is highly likely that forces with similar magnitude are applied from closeto-symmetrical directions. This creates the effect of deadlock similar to the case of two individuals in Figure 3. When one character releases the object, the deadlock is broken and the applied forces become asymmetrical. This re-enforces the probability that individuals who are pulling from opposing direction reposition themselves. The final result is the reorganised formation of the individual spacing. When a character approaches the target position and finds it occupied by another agent, it attempts alternative target locations until it finds an available one.

\subsection{Adapting to Different Numbers of Individuals}

One of the advantages of the decentralised paradigm is the scalability to different numbers of individuals. This is validated in our work by simulating the task of transport with different group sizes (Figure 5). In the real world, there always exists an optimal group size in order to balance between transport speed and energy efficiency. A larger group would recruit more individuals and thus increase the transport speed. However, the transport speed may not increase linearly with the number of individuals. Figure 6 plots the average transport speed with respect to the number of individuals. The results show that the linear relationship only exists for small team 459 sizes ( $2 \sim 3$ individuals). For greater numbers of 460 individuals, the speed increases at a slower rate. 461 Based on our simulation observations, the rea- 462 sons for such a nonlinear relationship are two 463 fold. First, when more individuals form a group, $\quad 464$ the object is generally transported at a higher 465 speed, which in turn increases the probability of 466 individuals repositioning themselves to different $\quad{ }_{467}$ attachment points (Equation 3). Second, for an 468 object with a fixed geometric size, an increasing 469 number of individuals would have difficulty in 470 finding an appropriate attachment position and 471 avoiding bodily collisions with existing individ- 472 uals who are already attached to the object. This 473 leads to the fact that a significant proportion of 474 additional individuals' time would then be spent 475 on looking for an attachment point instead of ac- 476 tually pulling the object.

\section{7}

\subsection{Following a Curve}

478

In the previous examples, individuals know the 479 location of the destination (or the nest). Forces 480 are applied as vectors from their current location $\quad 481$ towards the final destination. In the real world, 482 ants determine their path back to the nest via 483 pheromone trails, which are chemicals laid by ${ }_{484}$ nest members and strengthened as the transport 485 continues. Our method allows modelling com- 486 plex pheromone paths by user-defined curves. 487 The curve is first uniformly parameterised with 488 the value range of $[0,1]$. Users can specify the 489 desired transport velocity on different segments 490 of the curve. At time $t$, the desired location is 491 passed to the controller and our method com- 492 putes the control inputs for the individuals. The 493 capability of following complex trajectories ex- 494 tends to scenarios such as obstacle avoidance 495 (Figure 7). Although this is not the exactly the 496 same as real ants, it is sufficient and flexible 497 enough to allow artists to reproduce such a be- 498 haviour for virtual ants. 456 457 453 us 458 .

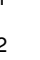
. .

.

0

.
" 


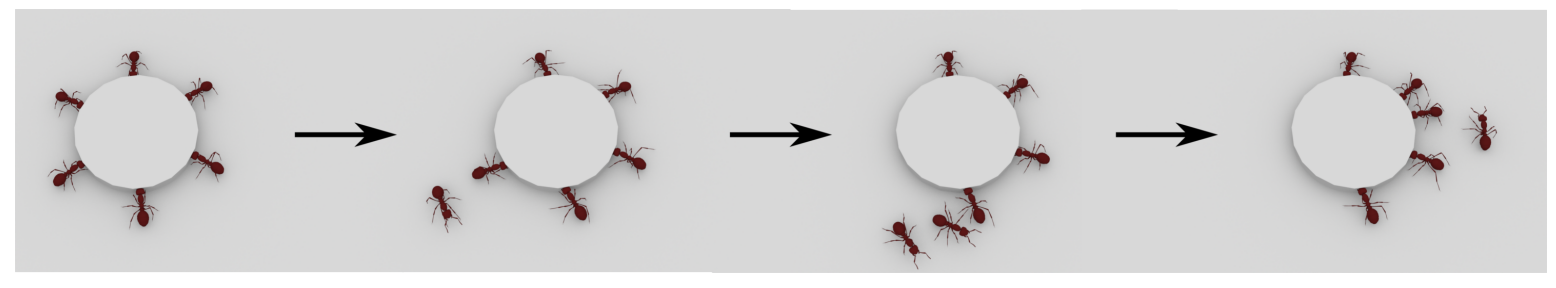

Figure 4: Collective transport by a team of six individuals. Characters are evenly distributed around the object during initialisation. As time proceeds, characters break the deadlock and start to move the object in an uncoordinated but collective fashion.

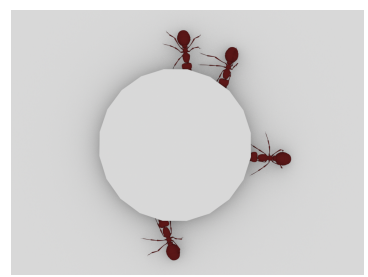

(a)

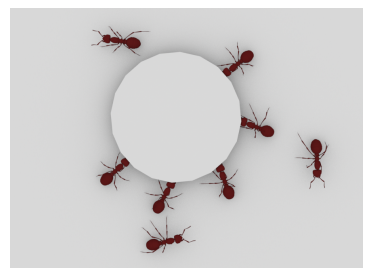

(b)

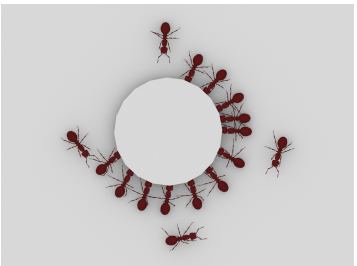

(c)

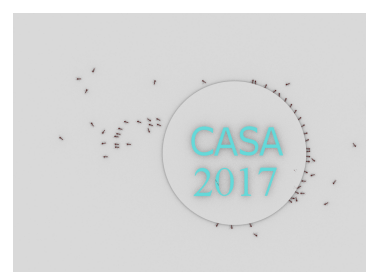

(d)

Figure 5: Simulating the task of collective transport with different numbers of individuals (from left to right: $4,8,16,60)$.

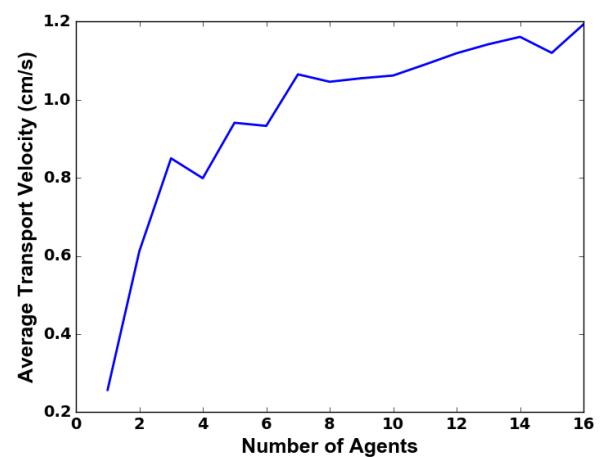

Figure 6: Average transport speed of an object with respect to the number of individuals.

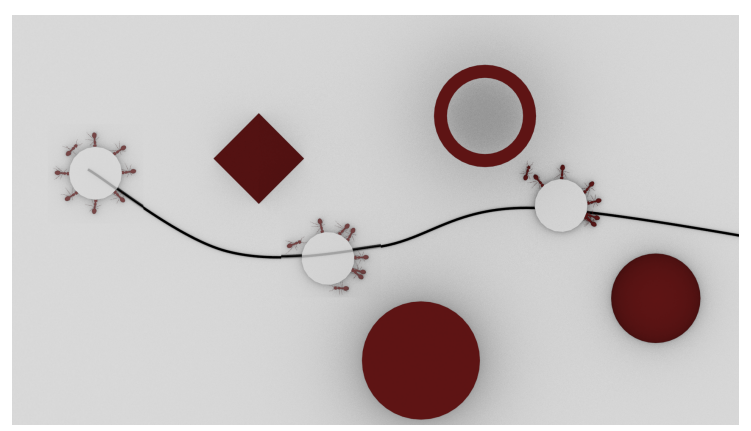

Figure 7: A group of ants are transporting an object along a predefined curve, creating the effect of obstacle avoidance.

\subsection{Adapting to Objects with Different Shapes}

Our method is also capable of simulating a 502 group of ants transporting objects of arbitrary 503 shape. This is validated in the example of the 504 demo of objects with text-shape (Figure 8). The 505 contour of the objects is represented as a set of 506 connected line segments, which are checked for 507 collisions with the geometry of the individual 508 ants.

\section{9}

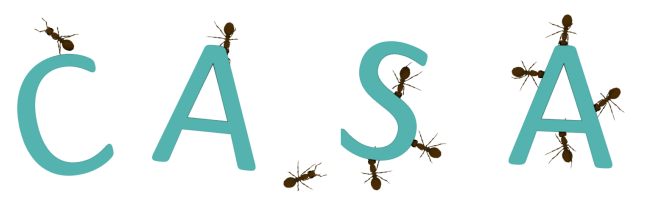

Figure 8: Ants transport objects of different shapes.

\subsection{External Intervention}

In the real world, the object could be abruptly 511 relocated to another location by wind or even 512 seized by competitors. We categorise such inci- 513 dences, which cause the sudden relocation of the 514 object, as external intervention. The stability of 515 our method is demonstrated when there exists an 516 external intervention during the process of trans- 517 
port. After the intervention is introduced, all individuals are forced to release the object. They then enter the state of search and start looking for the relocated object or an alternative if the original object is not found. Each individual switches to the state of approach and then transport if an object is detected within their sensory range. With this proposed strategy, the system is capable of accommodating external intervention (see Figure 9).

\section{Conclusions}

In a classical multi-character system, direct communication exists between individuals. The problem of stigmery, like the task of collective transport of ants, differentiates from classical systems because individuals act as if they are alone and do not directly share information with each other. This paper models the limited intelligence of real ants in nature and simulates the behaviour of collective transport which is commonly observed in ant colonies. This model is decentralised, scalable and does not require $a$ priori information about the prey object. With no explicit communication but only with individual local sensing, this method is able to scale to scenarios with different numbers of individuals.

One future direction for this work would be to further validate our model by comparing our simulation model with real ants. This would include capturing video footage of real ants involved in the task of collective transport. The relevant information, including the timing and positioning of group members, could then be extracted using techniques from computer vision. The comparison could be used to optimise the parameters used in our behaviour engine model. Another challenge that is not yet fully solved in our work is the design of the neural networks. The current architecture is constructed based on empirical knowledge. Since there is no universal guidance on the design of neural networks, and compared to the large possibility of network architectures, we can only approach the solution via limited experimentation. How to extend this controller to scenarios other than the task of collective transport is one of the future directions for this research.

\section{References}

[1] J. H. Sudd, "The transport of prey by ants," 567 Behaviour, vol. 25, no. 3/4, pp. 234-271, 568 Jan. 1965, ArticleType: research-article / 569 Full publication date: 1965 / Copyright 570 1965 BRILL.

[2] N. R. Franks, "Teams in social insects: 572 group retrieval of prey by army ants 573 (eciton burchelli, hymenoptera: Formici- 574 dae)," Behavioral Ecology and Sociobiol- 575 ogy, vol. 18, no. 6, pp. 425-429, May 576 1986.

[3] S. Berman, Q. Lindsey, M. S. Sakar, V. Ku- 578 mar, and S. Pratt, "Experimental study and 579 modeling of group retrieval in ants as an 580 approach to collective transport in swarm 581 robotic systems," Proceedings of the IEEE, 582 vol. 99, no. 9, pp. 1470-1481, 2011.

[4] K. Hyun, K. Lee, and J. Lee, "Mo- 584 tion grammars for character animation," in 585 Computer Graphics Forum, vol. 35, no. 2. 586 Wiley Online Library, 2016, pp. 103-113. 587

[5] J. C. Chan, J. K. Tang, and H. Le- 588 ung, "Synthesizing two-character interac- 589 tions by merging captured interaction sam- 590 ples with their spacetime relationships," in 591 Computer Graphics Forum, vol. 32, no. 7. 592 Wiley Online Library, 2013, pp. 41-50. 593

[6] J. Hwang, I. Suh, and T. Kwon, "Edit- 594 ing and synthesizing two-character mo- 595 tions using a coupled inverted pendulum 596 model," in Computer Graphics Forum, 597 vol. 33, no. 7. Wiley Online Library, 598 2014, pp. 21-30.

[7] K. Wampler, E. Andersen, E. Herbst, 600 Y. Lee, and Z. Popovic, "Character anima- 601 tion in two-player adversarial games," Acm 602 Transactions on Graphics, vol. 29, no. 3, 603 pp. 483-496, 2010.

[8] H. P. Shum, T. Komura, and S. Ya- 605 mazaki, "Simulating multiple character in- 606 teractions with collaborative and adversar- 607 ial goals," IEEE Transactions on Visual- 608 ization and Computer Graphics, vol. 18, 609 no. 5, pp. 741-752, 2012.

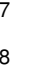

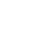

3
4
5
77

.

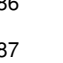
. .

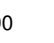

\section{(2)}

\section{.}

.

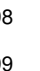




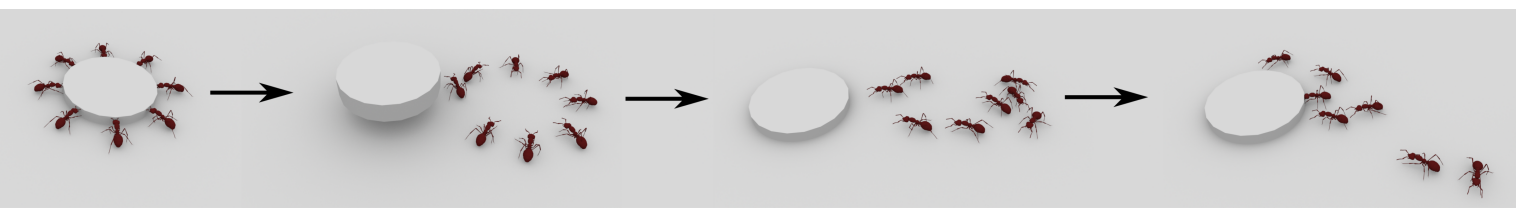

Figure 9: During the process of transport, user intervention can be introduced to abruptly move the object to another location. In such cases, group members return to searching for the object and rearrange themselves to form a new collective system to continue the transport task.

[9] J. Won, K. Lee, C. O'Sullivan, J. K. Hodgins, and J. Lee, "Generating and ranking diverse multi-character interactions," ACM Transactions on Graphics (TOG), vol. 33, no. 6, p. 219, 2014.

[10] Q. Gu and Z. Deng, "Generating freestyle group formations in agent-based crowd simulations," IEEE computer graphics and applications, vol. 33, no. 1, pp. 20-31, 2013.

[11] J. Henry, H. P. Shum, and T. Komura, "Interactive formation control in complex environments," IEEE transactions on visualization and computer graphics, vol. 20, no. 2, pp. 211-222, 2014.

[12] L. He, J. Pan, S. Narang, and D. Manocha, "Dynamic group behaviors for interactive crowd simulation," in Proceedings of the ACM SIGGRAPH/Eurographics Symposium on Computer Animation, ser. SCA '16. Aire-la-Ville, Switzerland, Switzerland: Eurographics Association, 2016, pp. 139-147.

[13] C. R. Kube and E. Bonabeau, "Cooperative transport by ants and robots," Robotics and autonomous systems, vol. 30, p. 85101, 2000.

[14] S. Patil, J. Van Den Berg, S. Curtis, M. C. Lin, and D. Manocha, "Directing crowd simulations using navigation fields," IEEE Transactions on Visualization and Computer Graphics, vol. 17, no. 2, pp. 244254, 2011.

[15] S. Takahashi, K. Yoshida, T. Kwon, K. H. Lee, J. Lee, and S. Y. Shin, "Spectralbased group formation control," in Computer Graphics Forum, vol. 28, no. 2. Wiley Online Library, 2009, pp. 639-648.
[16] A. Loria, J. Dasdemir, and N. A. Jarquin, 649 "Leader-follower formation and tracking 650 control of mobile robots along straight 651 paths," IEEE Transactions on Control Sys- 652 tems Technology, vol. 24, no. 2, pp. 727- 653 $732,2016$.

[17] M. Rubenstein, A. Cabrera, J. Werfel, 655 G. Habibi, J. McLurkin, and R. Nag- 656 pal, "Collective transport of complex ob- 657 jects by simple robots: theory and ex- 658 periments," in Proceedings of the 2013659 international conference on Autonomous 660 agents and multi-agent systems, ser. AA- 661 MAS '13. Richland, SC: International 662 Foundation for Autonomous Agents and 663 Multiagent Systems, 2013, p. 4754.

[18] J. H. Sudd, "The transport of prey by an 665 ant, pheidole crassinoda em." Behaviour, ${ }_{666}$ vol. 16, no. 3/4, pp. 295-308, Jan. 1960, 667 ArticleType: research-article / Full pub- ${ }_{668}$ lication date: 1960 / Copyright 1960669 BRILL.

[19] S. Guo, J. Chang, X. Yang, W. Wang, and 671 J. Zhang, "Locomotion skills for insects 672 with sample-based controller," Computer 673 Graphics Forum, vol. 33, no. 7, pp. 31-40, 674 2014.

[20] S. Guo, J. Chang, Y. Cao, and J. Zhang, "A 676 novel locomotion synthesis and optimisa- 677 tion framework for insects," Computers \& 678 Graphics, vol. 38, pp. 78-85, 2014.

[21] Y. Jia, E. Shelhamer, J. Donahue, 680 S. Karayev, J. Long, R. Girshick, 681 S. Guadarrama, and T. Darrell, "Caffe: 682 Convolutional architecture for fast fea- 683 ture embedding," in Proceedings of the 684 22nd ACM international conference on 685 Multimedia. ACM, 2014, pp. 675-678. 686 